\title{
The Catalina Real-time Transient Survey
}

\author{
A. J. Drake ${ }^{1}$, S. G. Djorgovski ${ }^{1,6}$, A. Mahabal $^{1}$, J. L. Prieto ${ }^{2}$, \\ E. Beshore ${ }^{4}$, M. J. Graham ${ }^{1}$, M. Catalan ${ }^{3}$, S. Larson ${ }^{4}$, \\ E. Christensen ${ }^{5}$, C. Donalek ${ }^{1}$ and R. Williams ${ }^{1}$ \\ ${ }^{1}$ California Institute of Technology, Pasadena, CA 91125, USA. \\ email: ajd@cacr.caltech.edu \\ ${ }^{2}$ Dept. of Astrophysical Scinces, Princeton University, NJ 08544, USA. \\ ${ }^{3}$ Depto. de Astronomia y Astrofisica, Pont. Uni. Catolica de Chile, Santiago, Chile. \\ ${ }^{4}$ Lunar and Planetary Lab, University of Arizona, Tucson, AZ 85721, USA. \\ ${ }^{5}$ Gemini South Observatory, c/o AURA, Casilla 603, La Serena, Chile. \\ ${ }^{6}$ Distinguished Visiting Professor, King Abdulaziz Univ., Jeddah, Saudi Arabia.
}

\begin{abstract}
The Catalina Real-time Transient Survey (CRTS) currently covers $33,000 \mathrm{deg}^{2}$ of the sky in search of transient astrophysical events, with time base-lines ranging from 10 minutes to $\sim 7$ years. Data provided by the Catalina Sky Survey provide an unequalled base-line against which $>4,000$ unique optical transient events have been discovered and openly published in real-time. Here we highlight some of the discoveries of CRTS.
\end{abstract}

Keywords. (stars:) supernovæ: general, (galaxies:) BL Lacertae objects: general, stars: dwarf novæ stars, stars: flare, galaxies: dwarf

\section{Introduction}

For the past four years the Catalina Real-time Transient Survey (CRTS; Drake et al. 2009a, Djorgovski et al. 2011, Mahabal et al. 2011) has systematically surveyed tens of thousands of square degrees of the sky for transient astrophysical events. CRTS discovers highly variable and transient objects in real-time, making all discoveries public immediately, thus benefiting a broad astronomical community. Data are leveraged from three telescopes, operated by LPL in a search for NEOs; they cover up to $\sim 2,500 \mathrm{deg}^{2} \mathrm{per}$ night with four exposures separated by $\sim 10 \mathrm{mins}$. The total survey area is $\sim 33,000 \mathrm{deg}^{2}$ and reaches depth $V \sim 19$ to $21.5 \mathrm{mag}$ (depending on the telescope) during 23 nights per lunation. All data are automatically processed as they are taken, and optical transients (OTs) are immediately distributed using a variety of electronic mechanisms (see http://www.skyalert.org/ and http://crts.caltech.edu/). CRTS has so far discovered $>4,000$ unique OTs including $>1,000$ supernovæ and 500 dwarf novæ.

\section{Discoveries}

Supernovæ and their hosts. Supernovæ are both cosmological tools and probes of the final states of stellar evolution. While many astronomical surveys focus on Type Ia SNe (being standard candles), CRTS uses its wide-area coverage to look for rare types of events that may be missed by many traditional SN surveys. With $>1,000$ SNe (CRTS published more SN discoveries in both 2009 and 2010 than any other survey), this data set has allowed us to carry out a systematic exploration of supernovæ properties, leading to the discovery of extremely luminous supernovæ and supernovæ in extremely faint host galaxies, with $M_{V} \sim-12$ to -13 , i.e., $\sim 0.1 \%$ of $L_{*}$. 

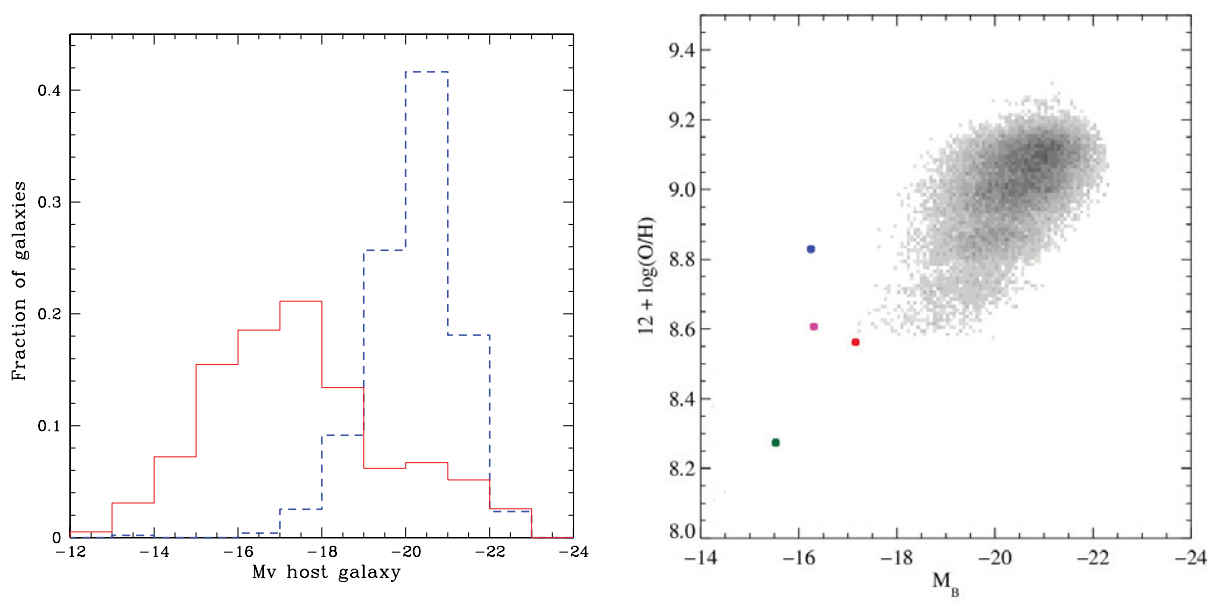

Figure 1. SN Hosts. Left: A comparison of supernovæ host-galaxy absolute magnitudes. Solid line: CRTS. Short-dashed line the Lick Observatory Supernovæ Search (LOSS). Right: Host luminosity and metallicity for four energetic Type IIn SN host galaxies, compared with 53,000 star-forming galaxies from SDSS (Tremonti et al. 2004).

Two especially interesting classes of luminous SNe discovered by CRTS, ROTSE and PTF include luminous Type Ic SNe: SN 2005ap (Quimby et al. 2007), SN 2009de (Drake et al. 2009b, 2010), SN 2009jh (Drake et al. 2009, Quimby et al. 2011), SN 2010gx (Mahabal et al. 2010; Pastorello et al. 2010a, 2010b), and CSS110406:135058+261642 (Drake et al. 2011b) and ultra-luminous and energetic Type IIn SNe: SN 2008fz, SN 2009jg, etc. (Drake et al. 2009c, 2010, 2011a). These supernovæ have been found to favour extremely faint host galaxies (Drake et al. 2009a, 2010), suggesting the importance of host-galaxy environment and explaining why more such events have not been discovered previously. In Fig. 1 we contrast the SN host-galaxy absolute magnitudes (from CRTS) with those from the long-running Lick Observatory SN Search (LOSS; Filippenko et al. 2001) which concentrates on large nearby galaxies.

The rate of our SN discovery in intrinsically faint galaxies implies phenomenally high specific SN rates (Drake et al. 2009a). Although such galaxies are common, a very small fraction of all baryonic matter is expected in them (Kauffmann et al. 2003). Evidence suggests that those galaxies include blue compact dwarfs and irregular dwarfs, where excessive star formation rates accelerate $\mathrm{SNe}$ rates for the most rapidly evolving massive stars (progenitors of luminous SN). The presence of additional evidence for enhancements in SN Ia rates, of up to $1500 \%$, has been speculated by Della Valle \& Panagia (2003).

It is likely that these dwarf galaxies have low metallicities owing to a delayed onset of star formation and expulsion of enriched SN ejecta from their shallow potential wells. According to the galaxy mass-metallicity relationship (Tremonti et al. 2004), low-luminosity hosts are expected to be low-metallicity hosts. This prediction was recently confirmed in the work of Neill et al. (2011), Stoll et al. (2011) and Kozlowski et al. (2010) as well as in our recent work shown in Fig. 1. Low metallicities are speculated to lead to a top-heavy IMF, which would account both for an enhanced specific SN rate and for the propensity for highly luminous events (from high-mass progenitors). Low-metallicity host galaxies are also linked to the broad-line type-Ic hypernovæ associated with long-timescale GRBs (Stanek et al. 2006).

Another interesting discovery involves a new class of SNe that may be associated with AGN accretion disks. Possibly the most luminous and optically energetic SN ever 
discovered, CSS100217 (within the AGN disk of a bright NLS1 galaxy), demonstrates that extreme supernovæ can occur in a variety of extreme environments (Drake et al. 2011a).

Blazars. Highly variable optical and radio sources, blazars are often targeted for optical follow-up at other wavelengths after their outbursts. CRTS provides an unbiased, statistical optical monitoring of known blazar sources over $75 \%$ of the sky. Owing to the erratic nature of blazar variability and the association of those sources with previously catalogued, and often faint, radio sources, we have found several tens of likely blazars based in transient outburst events. We have also discovered variability-selected blazar counterparts to the previously unidentified FERMI gamma-ray sources. CRTS data are being combined with radio data from the Owens Valley Radio Observatory, and will be used to provide better constraints to the theoretical models of blazar emission and variability.

Dwarf Novæ and UV Ceti variables. CRTS has discovered more than 500 new dwarf-nova-type cataclysmic variables (CVs). Since those objects are found in real time, the outbursts are often followed up. Thus far, $132 \mathrm{CV}$ discovery alerts have been sent to users of the VSNET system (www.kusastro.kyoto-u.ac.jp/vsnet/), resulting in successful period determination in dozens of systems. Similarly, CRTS has discovered over 100 UV Ceti variables (flare stars), varying by several magnitudes within minutes. The rate of such flares is still poorly constrained, and must be understood so that future surveys can find rare types of rapid transients. The short cadence of CRTS is well tuned to the discovery of such events. Another class of rapid transients are eclipses of whitedwarf binary systems, which probe the end state of stellar binary evolution. Although first discovered in real time, archival searches have revealed dozens more such eclipsing systems, including some with low mass companions (Drake et al. 2011c).

\section{Acknowledgements}

CRTS is supported by the NSF grant AST-0909182. We thank the personnel of many observatories involved in the survey and the follow-up observations.

\section{References}

Della Valle M. \& Panagia, N. 2003, ApJ, 587, L71

Drake A. J., et al. 2009a, ApJ, 696, 87

Drake A. J., et al. 2009b, CBET, 1958

Drake A. J., et al. 2009c, CBET, 1766

Drake A. J., et al. 2010, ApJ, 718, 127

Drake A. J., et al. 2011a, APJ, 735, 106

Drake A. J., et al. 2011b, ATEL, 3343

Drake A. J., et al. 2011c, $A p J$, arXiv:1009.308

Djorgovski, S. G., et al. 2011, JAXA, in press, arXiv:1102.5004

Filippenko, A., Li, W. D., Treffers, R. R., \& Modjaz, M. 2001, PASP Conf Ser., 246, 121

Kauffmann G., et al. 2003, MNRAS, 341, 33

Kozlowski, S., et al. 2010, ApJ, 722, 1624

Mahabal, A. et al. 2009, ATEL, 1713, 1

Neill J., et al. 2011, ApJ, 727, 15

Pastorello, A. et al. 2010a, CBET, 2413

Pastorello, A. et al. 2010b, ApJ, 724, 16L

Quimby, R. et al. 2007, ApJ, 668, 99

Quimby, R. et al. 2011, NATURE, 474, 487

Stanek, K. Z., et al. 2006, AcA, 56, 333

Stoll, R., et al. 2011, ApJ, 730, 34

Tremonti, C. et al. 2014, ApJ, 613, 898 